\title{
Ultrasonic methods in determining elastic material properties of fibres in suspension
}

\author{
Torbjörn Löfqvist, Jan Niemi and Yvonne Aitomäki \\ Department of Computer Science and Electrical Engineering \\ Luleå University of Technology \\ 97187 Luleå, Sweden \\ Email: tlt@ltu.se
}

\begin{abstract}
The presented study concerns the application of the pulse-echo ultrasound measurement technique in determining the elastic properties of fibres suspended in water. The two kinds of fibre materials are investigated, nylon $6 / 6$ fibres and softwood pulp fibres. The fibre mass fraction was $0.5 \%$ for nylon and ranges from $0 \%$ up to $1 \%$ for softwood pulp. The ultrasonic measurements are performed in the frequency range of 2-11 MHz. It is shown that the velocity dispersion of the ultrasound is small for each suspension sample. In obtaining the fibres longitudinal Young's modulus two methods are used, one based on phase velocity and one based on acoustic attenuation. It is found that both methods gives reasonable estimates of the longitudinal Young's modulus for nylon 6/6. For pulp fibres the determined Young's modulus is overestimated in comparison with earlier findings.
\end{abstract}

\section{INTRODUCTION}

The modelling the attenuation and phase velocity of acoustic waves in particulate suspensions, where the dispersed phase consists of non-homogenous or non-spherical particles, has been a field of research for some decades but is still an area under development. Generally, the scattering and absorption of acoustic energy depends on the particles shape and size distribution, the constituent's thermophysical properties, their volume concentration and the frequencies of the acoustic wave.

The mechanisms behind attenuation and phase velocity in disperse systems of homogenous materials of spheres and droplets have been investigated by a number of researchers and is well known. When studying a suspension of a biological material, like paper pulp fibres or red blood cells, the scattering and absorbtion ultrasound is difficult to quantify because the biological material's thermophysical properties are often unknown. Furthermore, the suspended biological material will often also have a non-spherical geometry and a complex internal structure, such as layered cell walls, which possibly will affect the scattering and absorption of acoustic energy.

The compressibility of any fibre material is linked to the fibres elastic properties. The elastic properties are a few of the physical parameters that must be known when existing scattering theories are employed to model the attenuation of ultrasound in a paper pulp fibre suspension, as for example Habeger's [2]. One method to obtain the adiabatic compressibility is to use Urick's [1] equation, where the phase velocity is related to the mean density and the mean compressibility of the suspension. Urick's equation holds its virtues in its simplicity. However, this simplicity conceals an underlying, inherent complexity and the Urick equation can be shown to be only a special case of a more general descriptions, [3].

In this paper we compare two approaches in obtaining the elastic properties of fibres in suspension. Firstly, the experimentally obtained phase velocity is used togheter with Urick's equation in estimating the longitudinal Young's modulus in nylon and pulp fibres. Secondly, we use the experimentally obtained attenuation spectra in conjunction with a model, derived from first principles, for the attenuation in a fibre suspension in estimating the elastic parameters of the fibre material, i.e. solving the inverse problem.

\section{THEORY}

The velocity of sound in a dispersion is generally related to frequency, physical properties and volume fraction of each constituent. At the simplest level, the velocity of sound, $c$, can be expressed by the Urick equation [1],

$$
c=\frac{1}{\sqrt{\rho \kappa}}
$$

where $\rho$ and $\kappa$ are the suspension's density and adiabatic compressibility, respectively. Urick assumes that both density and adiabatic compressibility are additive properties of the corresponding quantities, each proportional to their respective volume concentration,

$$
\begin{gathered}
\rho=\rho_{s} \phi+\rho_{l}(1-\phi) \\
\kappa=\kappa_{s} \phi+\kappa_{l}(1-\phi)
\end{gathered}
$$

where $\rho_{s}, \kappa_{s}$ and $\rho_{l}, \kappa_{l}$ are the density and adiabatic compressibility of fibres and water respectively. Above, $\phi$ indicates the concentration by volume of the suspended fibres. Urick's equation considers the suspension as being a perfect mixture of two fluids and does not account for the particulate nature of the suspension. Urick's equation requires only a few bulk parameters to be known. This is advantageous in situations where the suspended particles have complicated shapes or when a detailed knowledge of all thermophysical parameters of the suspended material is difficult to obtain. Dividing equation (1) with the speed of sound for water, $c_{l}=1 / \sqrt{\rho_{l} \kappa_{l}}$, and combining equations (1)-(3), we obtain an expression for the adiabatic compressibility, $\kappa_{s}$, of the fibres 


$$
\kappa_{s}=\kappa_{l}\left[1-\frac{1}{\phi} \frac{1-\left(\frac{c_{l}}{c}\right)^{2}-\phi\left(1-\frac{\rho_{s}}{\rho_{l}}\right)}{1-\phi\left(1-\frac{\rho_{s}}{\rho_{l}}\right)}\right] .
$$

Thus, by measuring the velocity of sound in the suspension the compressibility of the fibre material could be determined. Generally, the velocity of sound in a suspension is frequency dependent. In order to retain the validity of equation (4) it is required that the velocity dispersion has to be small.

In the presented study two kinds of fibres are used, nylon $6 / 6$ fibres and paper pulp fibres. The nylon fibres has well known thermophysical properties and are all of straight, equal length, cylindrical shape. As a contrast, the pulp fibres has less known thermophysical properties and are distributed in both size, shape and also in their thermophysical properties.

The volume concentration, $\phi$, of nylon fibres is straightforward to calculate from fibre concentration by mass, $c_{m}$. For the pulp samples, the volume concentration of fibres is calculated from fibre concentration by mass using a relation derived by Wikström [4]. In his derivation, Wikström includes radial swelling of the fibre wall due to water absorption in the fibre material. From Wikström we get

$$
\phi=\frac{\pi d^{2} \rho_{l}}{4 C_{o}} \frac{c_{m}}{1+c_{m}\left(\frac{\rho_{l}}{\rho_{s}}-1\right)}
$$

where $d$ is the fibre diameter. The variable $C_{o}$ is named coarseness, or fibre weight value, and expresses the mean dry fibre mass per unit of fibre length. Since we are using mass fractions $c_{m} \leq 1 \%$, equation (5) is approximated for small values of $c_{m}$ as,

$$
\phi \simeq \frac{\pi d^{2} \rho_{l}}{4 C_{o}} c_{m} .
$$

By combining equations (4) and (6) we arrive at

$$
\kappa_{s}=\kappa_{l}\left[1-\frac{4 C_{o}}{\pi d^{2} \rho_{l} c_{m}} \frac{1-\left(\frac{c_{l}}{c}\right)^{2}-\frac{\pi d^{2} \rho_{l}}{4 C_{o}} c_{m}\left(1-\frac{\rho_{s}}{\rho_{l}}\right)}{1-\frac{\pi d^{2} \rho_{l}}{4 C_{o}} c_{m}\left(1-\frac{\rho_{s}}{\rho_{l}}\right)}\right] .
$$

The adiabatic compressibility is the inverse of the bulk modulus, $\kappa_{s}^{\prime}=1 / K_{s}$. To go beyond the bulk modulus some simplifying assumptions are necessary. It is assumed that the fibre material is visco-elastic with constant coefficients. Furthermore, we assume that the wavelength is larger than the diameter of the fibre. Also, we assume that the principal mode of wave propagation is longitudinal. It is known that the velocity of compression waves in solid materials with low attenuation $\left(\alpha_{s} \ll \omega / c_{s}\right)$ is

$$
c_{s}^{2}=\frac{1}{\rho_{s}}\left(K_{s}^{\prime}+\frac{4}{3} G_{s}^{\prime}\right)
$$

and if we only consider thin rod propagation

$$
c_{s}^{2}=\frac{E^{\prime}}{\rho_{s}} .
$$

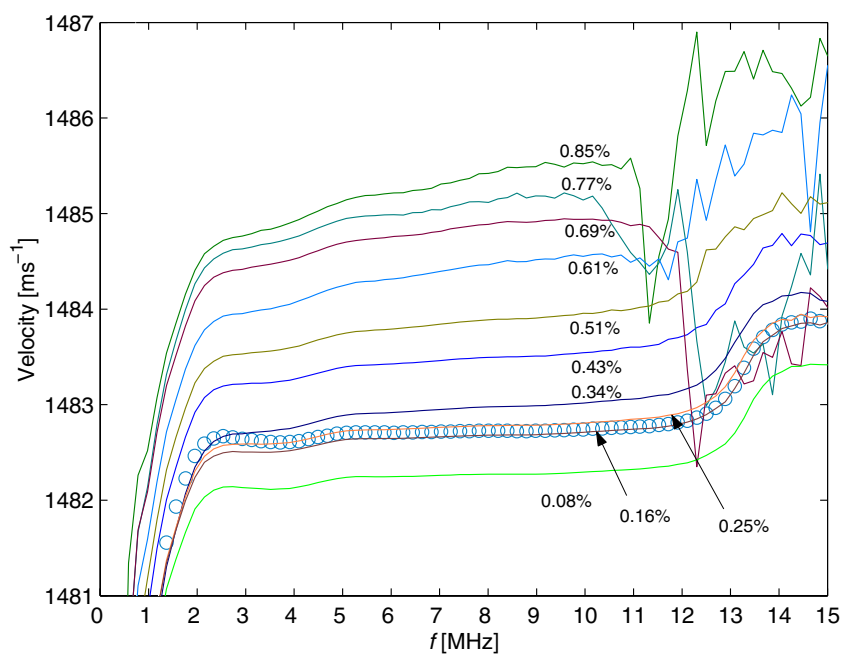

Fig. 1. Phase velocity spectra for the softwood pulp samples. The bandwidth is $2.3-10.5 \mathrm{MHz}$. The numbers adjacent to the curves represents fibre mass fractions of the samples.

In equation (8) and (9), $E^{\prime}$ is the elastic modulus, $K_{s}^{\prime}$ is the bulk modulus, $G_{s}^{\prime}$ is the adiabatic shear modulus and $c_{s}$ is the longitudinal wave velocity.

As a comparison we also consider an alternate way in determining the elastic parameters of the fibre material. In this case we use the experimentally obtained attenuation spectra in conjunction with a model for attenuation in a fibre suspension in estimating the parameters describing the fibre material, i.e. solving the inverse problem. The model, by Aitomäki [5], is based on an exact solution to the linearised energy, momentum and mass conservation equations for a straight, infinitely long, homogenous, circular cylinder immersed in a viscid fluid.

\section{EXPERIMENTAL TECHNIQUES}

The measurements of phase velocity and attenuation spectra are performed using a pulse-echo technique in a custom built measurement cell. Its construction and the measurement principles are described in detail in Löfqvist [6] and Niemi [7]. The theory of pulse-echo measurements of attenuation and sound speed can be found in McClements [8]. For this study a transducer with $10 \mathrm{MHz}$ center frequency, model V311, Panametrics, MA, USA, is used spanning a frequency range of $2-12 \mathrm{MHz}$, at $-10 \mathrm{~dB}$.

The nylon fibres used where supplied by Swissfloc, Emmenbrücke, Switzerland, and three fibre diameters where used, $17 \mu \mathrm{m}, 51 \mu \mathrm{m}$ and $55 \mu \mathrm{m}$. The length was $1.2 \mathrm{~mm}$ for the 17 $\mu \mathrm{m}$ fibre and $1.5 \mathrm{~mm}$ for the 51 and $55 \mu \mathrm{m}$ fibres.

The pulp used where sulphate processed, unbeaten, unfilled and unbleached softwood (70\% spruce and $30 \%$ pine) pulp supplied by a local kraftliner plant. The pulp stock was diluted with deionized and degassed water to mass fractions ranging from $0.1 \%$ to $1.0 \%$ in steps of $0.1 \%$.

Before and during each measurement series, the sample in the measurement cell was stirred to extract air bubbles and 


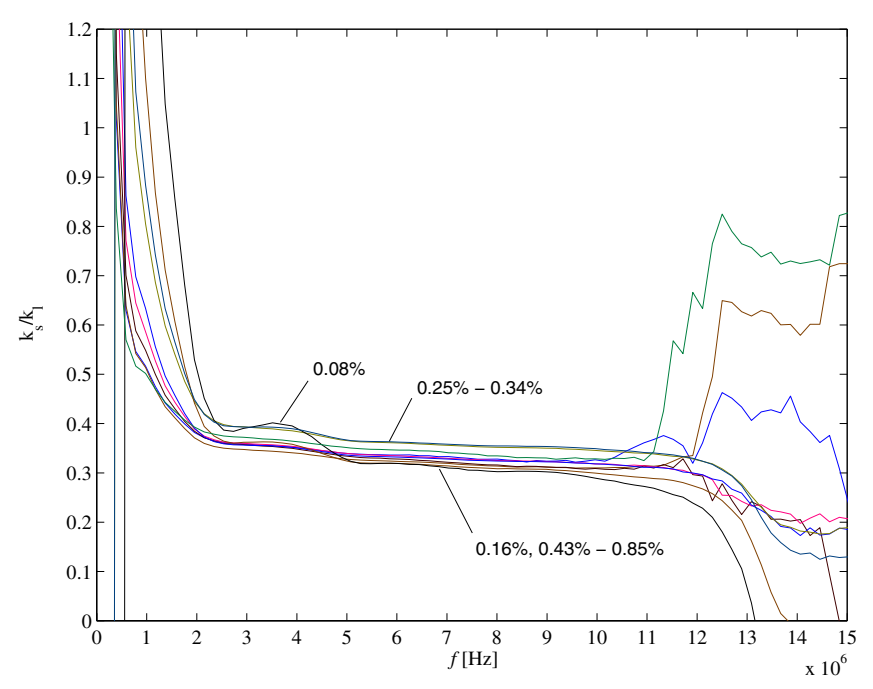

Fig. 2. Normalized compressibility for the softwood pulp samples. The fibre compressibility, $k_{s}$, has been normalized with the compressibility for pure water, $k_{l}$. The bandwidth is $2.3-10.5 \mathrm{MHz}$. The numbers adjacent to the curves represents fibre mass fractions of the samples.

keep fibers from sedimenting.

The thermophysical properties of nylon is well known and can be found in the literature. In this study we used the fibre density $\rho_{s}=1140 \mathrm{~kg} / \mathrm{m}^{3}$ for the nylon fibres. The fibre density for the softwood fibres is assumed to be $\rho_{s}=1500 \mathrm{~kg} / \mathrm{m}^{3}$, based on Ehrnrooth [9]. Fibre mass fraction, or consistency, of the pulp samples was determined after the tests, following the standardized procedure specified in [10]. Together with the cellulose in the pulp fibre material there is also lignin. The lignin content in the softwood pulp was $12.1 \%$. To characterize the pulp, the length weighted mean fibre lengths and coarseness values were determined using a Kajaani FS200 optical fibre analyzer, Valmet Oy, Finland.

\section{EXPERIMENTAL RESULTS}

The figure 1 show the phase velocity as a function of frequency for softwood pulp. As a reference, the phase velocity for pure water is included in the graph as a line of circles. The figure show that the velocity dispersion for each mass fraction is small, below $1 \mathrm{~m} / \mathrm{s}$ or less than $0.1 \%$. In figure 1 , the acoustic energy bandwidth is $\sim 2.3 \mathrm{MHz}$ to $\sim 12$ $\mathrm{MHz}$ for water and the smallest mass fractions of fibres. Since attenuation increases with increasing mass fraction, the highest relevant frequencies will decrease to $\sim 10.5-11 \mathrm{MHz}$ for the largest mass fractions. Using equation (7), the pulp fibre compressibility is calculated from phase velocity data for each sample. The calculated compressibility is subsequently normalized with tabulated values for the compressibility of water [11] and plotted as a function of frequency in figure 2. In figure 2 it is seen that the calculated fibre compressibility varies between the samples. However, the variation in compressibility stays within the experimental error. The small velocity dispersion seen in figure 1 appears as a small negative slope of the compressibility in graph 2 .

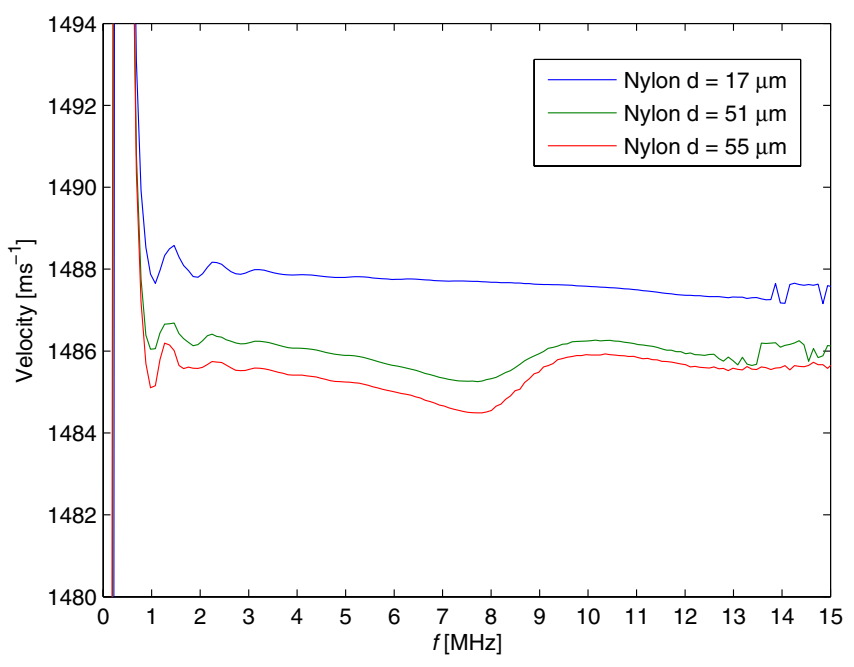

Fig. 3. Phase velocity spectra for the nylon fibre suspension samples. The bandwidth is $2.3-11 \mathrm{MHz}$.

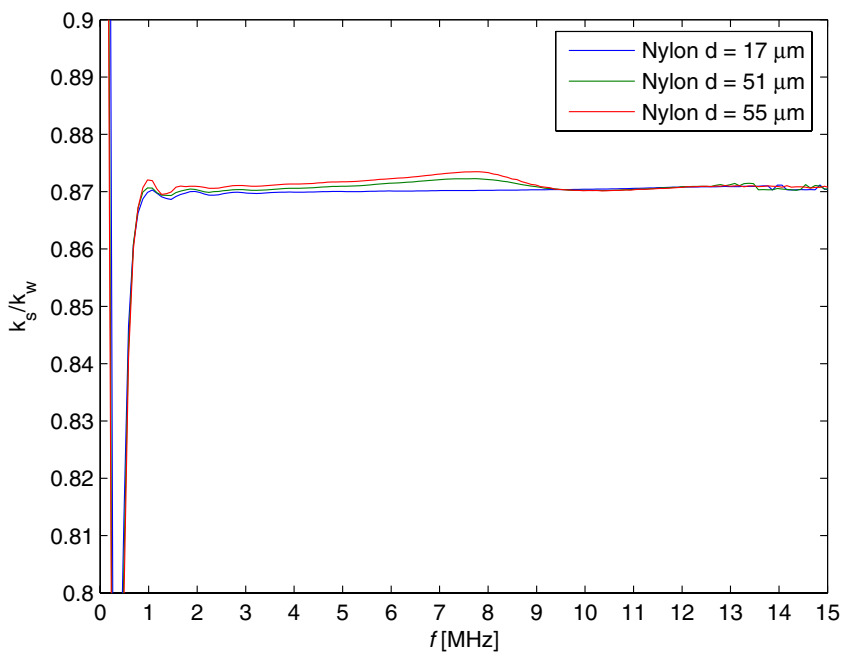

Fig. 4. Normalized compressibility for the nylon fibre suspension samples. The fibre compressibility, $k_{n}$, has been normalized with the compressibility for pure water, $k_{l}$. The bandwidth is $2.3-11 \mathrm{MHz}$

Using equation (4), the nylon fibre compressibility for all three samples are calculated from phase velocity data and normalized using the compressibility for pure water. The results are plotted in figure 4 . The figure show that the calculated compressibilities of the three nylon suspension samples falls onto the same curve. The effect of phase velocity dispersion appears to be small.

\section{A. Error analysis}

Since the uncertainties of the variables in the equation for the adiabatic compressibility of the fibres (7) are known, or are easily estimated, we can determine the uncertainty limit for the fibre compressibility $\kappa_{s}$ and the longitudinal Young's modulus $E^{\prime}$ by performing a general error propagation analysis 
TABLE I

MATERIAL DATA

\begin{tabular}{|c|c|c|c|}
\hline & & Softwood & Nylon $6 / 6$ \\
\hline Mean fibre & $\mathrm{d}[\mu \mathrm{m}]$ & $30 \pm 1$ & $17.32 \pm 0.54$ \\
\hline diameter $^{a}$ & & & $51.07 \pm 0.64$ \\
\hline & & & $55.17 \pm 0.91$ \\
\hline $\begin{array}{l}\text { Weighted mean } \\
\text { fibre length }\end{array}$ & $\mathrm{L}_{w}[\mathrm{~mm}]$ & $2.38 \pm 0.02$ & - \\
\hline Coarseness $^{b}$ & $\mathrm{C}_{o}[\mathrm{mg} / \mathrm{m}]$ & $0.259 \pm 0.002$ & - \\
\hline Density $^{c}$ & $\rho_{s}\left[\mathrm{Kg} / \mathrm{m}^{3}\right]$ & $1500 \pm 10$ & $1140 \pm 10$ \\
\hline Lignin content $^{b}$ & [\%] & 12.1 & - \\
\hline
\end{tabular}

on equation (7) above.

In the presented study, the fibre density was assumed to be $\rho_{s}=1500 \mathrm{~kg} / \mathrm{m}^{3}$ for the softwood fibres, based on Ehrnrooth [9] and $\rho_{s}=1140 \mathrm{~kg} / \mathrm{m}^{3}$ for nylon fibres. A way to improve the precision in determining the adiabatic compressibility would be to measure fibre densities in wet conditions more accurately.

\section{DISCUSSION}

The compressibility, which is the inverse of the bulk modulus, of pulp fibres is a parameter linked to the elastic properties of the fibre material. The elastic properties are some of the physical parameters that must be known when scattering theory, such as Habeger's [2], is employed to model the attenuation of ultrasound in a paper pulp fibre suspension.

The measured compressibility can be used to estimate the speed of sound in wet fibre material. Using a tabled value for the adiabatic compressibility of water $\kappa_{l}=4.878 \cdot 10^{-10}$ $m^{2} / N$, from Kaye and Laby [11], we find that the adiabatic compressibility for softwood is $\kappa_{s_{-} s o f t}=(1.56 \pm 0.07) \cdot 10^{-10}$ $\mathrm{m}^{2} / N$. The corresponding adiabatic compressibility for nylon is found to be $\kappa_{s_{-} n y l o n}=(4.25 \pm 0.02) \cdot 10^{-10} \mathrm{~m}^{2} / N$.

Neglect the adiabatic shear modulus in equation (8). Using the values on the compressibilities above, we then find the longitudinal wave speed in wet softwood fibres $c_{\text {soft }}=2067 \pm 53$ $\mathrm{m} / \mathrm{s}$ corrsesponding to an longitudinal Young's modulus of $E^{\prime}=6.4 \pm 0.37 \mathrm{GPa}$. To compare the Young's modulus determined above, we make use of data reported in Ehrnrooth [9]. Ehrnrooth experimentally determined the Young's modulus $E^{\prime}$ on single, wet spruce fibres (softwood) for different degrees of deligninfication of the fibres. Using data from Ehrnrooth we obtain for our case $E_{\text {wet_soft }}^{\prime} \simeq 4.4 \cdot 10^{9} \mathrm{~N} / \mathrm{m}^{2}$.

The resulting Young's modulus obtained from solving the inverse problem - using the attenuation spectra - as well as the results above are displayed in table 2 . The results for the nylon fibre suspensions showed that the fibres longitudinal Young's modulus depends on the diameter of the fibre, the lower value belongs to the $17 \mu \mathrm{m}$ fibre and the largest value to the 55 $\mu \mathrm{m}$ fibre. A possible explanantion for this is the difference in draw ratio, i.e. the applied stress that aligns the molcules in the axial direction of the fibre, when the fibres are manufactured. A higher degree of aligment increases the longitudinal Young's
TABLE II

COMPARISON OF LONGITUDINAL YOUNG'S MODULUS

\begin{tabular}{cccc}
\hline & $\begin{array}{c}\text { E' [GPa] } \\
\text { (literature) }\end{array}$ & $\begin{array}{c}\text { E' [GPa] } \\
\text { (phase velocity) }\end{array}$ & $\begin{array}{c}\text { E' [GPa] } \\
\text { (attenuation) }\end{array}$ \\
\hline Nylon 6/6 & $1.66-5^{a}$ & $2.35 \pm 0.08$ & $2.07-2.62 \pm 0.14$ \\
Softwood & $4.4^{b}$ & $6.4 \pm 0.37$ & - \\
\hline Data in the rightmost and middle column are based on experimentally \\
obtained attenuation and phase velocity spectra. Data in the \\
leftmost column are tabled data from a) manufacturer b) from [9].
\end{tabular}

modulus. When solving the inverse problem, which is illposed, the usage of phase velocity data could be beneficial since it adds additional information to the inversion process. At present, the attenuation spectra for the pulp samples has not been analyzed.

\section{Conclusions}

In the presented study, it is shown that a low intensity ultrasonic measurement technique could in principle be used to determine the longitudinal Young's modulus for a fibrous material in suspension. Two different fibre materials has been investigated, softwood (pine and spruce) and nylon 6/6. It is found that both methods give reasonable estimates on the longitudinal Young's modulus for nylon 6/6 fibres. The results for softwood pulp fibres differ to those obtained by other investigators. This could be due to the simplifications used in this study but it could also be due to the very limited amount of published reference values for water saturated fibres. These open issues calls for further investigations.

\section{REFERENCES}

[1] Urick R.J., "A sound velocity method for determining the compressibility of finely divided substances," J. Appl. Phys. 18, 983-987 (1947).

[2] Habeger C.C., "The attenuation of ultrasound in dilute polymeric fiber suspensions," J.Acoust. Soc. Am. 72, 870-878 (1982).

[3] Pinfield V.J., Povey M.J.W., Dickinson E., "The application of modified forms of the Urick equation to the interpretation of ultrasound velocity in scattering systems," Ultrasonics 33, 243-251 (1995).

[4] Wikström T., Flow of pulp suspensions at low shear rates, Licentiate thesis, Dept. of Chemical Engineering Design, Chalmers University of Technology, Göteborg, Sweden (1998).

[5] Aitomäki Y., Löfqvist T., "Estimating suspended fibre material properties by modelling ultrasound attenuation, Mathematical Modeling of Wave Phenomena, in AIP Conf. Proc. 834 American Institute of Physics, 2005, pp. 250-259.

[6] Löfqvist T., "Ultrasonic wave attenuation and phase velocity in a paper-fibre suspensions," 1997 IEEE Ultrasonics Symp. Proc., Toronto, Canada, 841-844, (1997) (IEEE, New York, 1997).

[7] Niemi J., Aitomäki Y., Lfqvist T.,"Ultrasonic measurements and modelling of attenuation and phase velocity in pulp suspensions," 2005 IEEE Ultrasonics Symp. Proc., Rotterdam, Netherlands, 775-779, (2005) (IEEE, New York, 2005).

[8] McClements D.J., Fairley P., "Ultrasonic pulse echo reflectometer" Ultrasonics 29, 403-405 (1991).

[9] Ehrnrooth E.M.L., Softening and mechanical behaviour of single wood pulp fibres-The influence of matrix composition and chemical and physical characteristics, PhD. Thesis, Dept. of Wood and Polymer Chemistry, University of Helsinki, Helsinki, Finland, 1982.

[10] SCAN-C 17:64, Scandinavian pulp, paper and board testing committee, 1964.

[11] Kaye G.W.C. and Laby T.H., Tables of physical and chemical constants, 14th ed., Longman Group Ltd., London, 1973. 